\title{
Asian Session
}

\author{
Date: November 10th (Fri) $8: 45 \sim 10: 45$ \\ Venue: Room 5 (福岡国際会議場 4F 413+414 会議室) \\ Chair : The Catholic Univ. \\ Seong Taek Oh \\ Hiroshima University Hospital Shiro Oka
}

\section{Session 1 : How to manage lateral spreading tumor}

\author{
AS1-1 Strategy of surveillance after EMR/ESD \\ Daehang Hospital \\ Eun-Jung Lee
}

The term "laterally spreading tumors (LSTs)" refers to the lateral growth of lesions at least $10 \mathrm{~mm}$ in diameter. LST should be further distinguished based on their granular or nongranular, homogenous or nonhomogenous appearance. Their relationship to the categories of the Paris classification is often a source of confusion. Some LST lesions can be classified as nonpolypoid, in the subtypes 0-IIa or 0-IIa+IIc. Other LST lesions associate with a polypoid (0-Is) or a nonpolypoid pattern. Some nongranular subtypes of LST are called pseudodepressed, to underline the distinction from simple depressed 0-IIc lesions.

Most post-polypectomy surveillance guidelines recommend stratifying the patients into low and high risk for longer or shorter surveillance intervals. The term 'high-risk adenomas' (or advanced adenomas) has usually been applied to adenomas that have high-grade dysplasia, or are $\geq 1 \mathrm{~cm}$ in size, or have $\geq 25 \%$ villous components, or are $\geq 3$ in number. 'Low-risks adenomas' (non-advanced adenomas) have none of these features. The surveillance strategy of 'high-risk adenomas' can be applied to LSTs.

\section{AS1-2 Tips for Colorectal ESD}

Department of Coloproctology, Aizu Medical Center Fukushima Medical University, Japan Kazutomo Togashi, Daiki Nemoto, Masato Aizawa

With the development of new devices and procedural standardization, colorectal endoscopic submucosal dissection (ESD) has made remarkable progress. Nevertheless, ESD remains technically difficult. We conducted a retrospective review evaluating 132 consecutive lesions (mean size $38.1 \mathrm{~mm}$, range 20-185) between April 2013 and September 2016 in a single center. Failure to resect en bloc occurred for 5 (4\%) lesions. Intra-procedural perforation occurred in $5(4 \%)$ patients, all of whom were treated successfully by endoscopic clipping. No delayed perforations occurred. Including slow dissection speed (lowest centile, $<8.5 \mathrm{~mm}^{2 / \mathrm{min}}$ ), difficult ESD was encountered in 18 lesions (14\%). Using both univariate and multivariate analyses, severe fibrosis and poor control of the colonoscope were significant predictors of difficult ESD. Improvements in colorectal ESD were evident, but lesion-specific factors (fibrosis) or technical factors (instrument control) remain obstacles to successful ESD. To overcome fibrosis in the submucosal layer, the pocket creation method and traction method using clips are useful. In case of poor control of the colonoscope, balloon-assisted colonoscopy should be applicable. Tips for overcoming difficult ESD based upon our experience are presented in this lecture.

\section{AS1-3 ESD for rectal neoplasms}

National Cancer Center, Korea

Chang Won Hong

Because screening colonscopies have been widely performed recently, many colorectal tumors in variable size have been diagnosed at precancerous or early cancer stage. Endoscopic resection is a very useful method of treatment for early colorectal neoplasms that have a negligible risk of lymph node metastasis. Endoscopic mucosal resection (EMR) is a useful therapeutic technique for colorectal tumors. However, for large tumors (esp. over $2 \mathrm{~cm}$ in diameter), the chance of piecemeal resection is high. If the tumor is resected in 
piecemeal, precise pathological evaluation (esp. depth, margin) is difficult and local recurrence rates are very high (5-45\%). Endoscopic submucosal dissection (ESD) was first developed in Japan for the treatment of early gastric and esophageal cancer. Several studies have shown the technical feasibility and clinical success of ESD for colorectal neoplasms allows en bloc resection but ESD experience is still limited in expert endoscopists for its technical difficulty.

\section{Indication of ESD for rectal neoplasm}

Many gastrointestinal societies state that the majority of rectal superficial lesions can be effectively removed in a curative way by EMR. ESD can be considered for treatment of rectal lesions with high suspicion of limited submucosal invasion that is based on two main criteria of depressed morphology and irregular or nongranular surface pattern, particularly if the lesions are larger than $20 \mathrm{~mm}$; or ESD can be considered for colorectal lesions with submucosal fibrosis.

- Lesions for which endoscopic en bloc resection is required

1) Lesions for which en bloc resection with snare EMR is difficult to apply

particularly LST-NG (PD) type

Lesions showing a Vi pit pattern

Carcinoma with shallow T1 (SM) invasion

Large depressed-type tumors

Large protruded-type lesions suspected to be carcinoma

2) Mucosal tumors with submucosal fibrosis

3) Sporadic localized tumors in conditions of chronic inflammation such as ulcerative colitis

4) Local residual or recurrent early carcinomas after endoscopic resection

\section{Complication of ESD for rectal neoplasm}

Main complications during ESD procedure are perforation and bleeding. Perforation during rectal ESD may cause the condition as focal emphysema in perirectal adipose tissue or mediastinal emphysema or subcutaneous emphysema. However, the presence of free air is not usually detected on $\mathrm{X}$-ray examination as a result of anatomical features.

There is no solid definitions on bleeding. Various definitions have been proposed such as a drop of hemoglobin by $>2 \mathrm{mg} / \mathrm{dL}$ or the requirement for transfusion. The presence of marked bloody stool after procedure or the requirement for a certain treatment for hemostasis is often defined as delayed bleeding.

A systematic review published in 2012 showed an adequate safety profile for colorectal ESD. The risk of post-ESD complication-related surgery (1\%) was reported to be negligible compared with the high efficacy of this procedure.

\section{Surveillance after ESD for rectal neoplasm}

All the retrospective studies evaluating the oncologic outcomes have shown that ESD is associated with lower local recurrence rates, ranging from 0-3\%, as compared with EMR, whose local recurrence rates vary from $0-9.1 \%$ for en bloc resections, and from $10-23.5 \%$ after piecemeal procedures. But, there is no clear evidence to inform decisions on optimal post-ESD surveillance. Therefore, most authors recommend surveillance sigmoidoscopy 3 to 6 months after resection in order to verify complete removal. If technical completeness is confirmed, total colonoscopy at 1 year after this reassessment is suggested, to check for local recurrence. However, no clear consensus has been reached on the actual method and time of surveillance.

\section{AS1-4 Newly developed EMR/ESD instruments}

Division of Gastroenterology and Heptaology, Taichung Veterans General Hospital ${ }^{1)}$, Department of Gastorenterology and Hepatology, Kindai University Faculty of Medicine ${ }^{2)}$ $\mathrm{Sz}^{-}$-Iuan Shiu ${ }^{1)}$, Hiroshi Kashida ${ }^{2}$ 


\title{
AS1-5 ESD for early colorectal neoplasms-Current status and controversies
}

\section{National Taiwan University \& Hospital}

Han-Mo Chiu

Detection and resection of colorectal adenoma has proven effective in reducing both colorectal cancer (CRC) incidence and mortality. Diagnosing CRC at early stage has higher likelihood of life-year saving or even cure. Endoluminal resection of carcinoma in situ, superficially invasive cancer or large colorectal adenoma can absolve the need of colorectomy or colonostomy and substantially improve quality of life of the patients after the treatment.

Piecemeal resection of large neoplasm has higher risk of recurrence and sometimes hinders comprehensive pathological analysis because of fragmentation of the specimen and cauterization effect. Endoscopic submucosal dissection (ESD), which is nowadays the standard way to treat early esophageal or gastric cancers, is also increasingly applied to treat colorectal neoplasms because it has significantly higher en bloc resection rate for neoplasms sized greater than $20 \mathrm{~mm}$. Nevertheless, distinct from esophageal and gastric neoplasms, the majorities of colorectal neoplasms are benign and can be treated with repeated endoluminal resection with endoscopic mucosal resection (EMR), which is much quicker and easier than ESD, in case residual neoplasms were founded at surveillance colonoscopy. In clinical practice, however, some specific neoplasms are justified to resect with ESD. Laterally spreading tumor (LST) is the major indication of ESD and its subtype non-granular type with pseudo-depression (LST-NG-PG) carries much higher risk of invasive cancer (more than 20\%) than other subtypes therefore is justified to resect en bloc with ESD. Other subtype such as mixed-nodular granular type LST (LST-G-MN) also has higher risk of invasive cancer at its nodular part and en bloc resection is also highly recommended.

According to previous study, T1 CRC carries 10\% risk of regional lymph node metastasis (LNM) therefore it is indispensible to ensure that lesion has minimal risk of LNM, which is closely correlated with some histological findings such as invasion depth, lymphovascular invasion, and budding. Accordingly endoscopists should be able to diagnose cancerous change or invasion depth accurately.

Current studies on ESD or EMR are mainly focusing on short-term outcomes such as en bloc resection rate, T1 resection rate, complication rate, or recurrence rate within subsequent few years. From oncological perspectives, it is, however, indispensible to obtain the long-term outcome in terms of cancerous recurrence or disease-free survival before it can be justified as the standard procedure to treat early CRC. The long-procedure time, higher invasiveness, higher cost and long learning curve are some of the issues that are continuously debated. Further studies are mandatory.

\author{
Chair : Chungnam National Univ. \\ St. Marianna University School of Medicine Toyoko Hospital \\ Commentator : Chungnam National Univ.
}

Woo Yong Lee

Tomohisa Furuhata

Ji Yeon Kim

\section{Session 2 : How to manage for rectal cancer following neoadjuvant chemoradiotherapy}

\section{AS2-1 How to evaluate the chemoradiotherapy response? \\ Department of Surgery, Yonsei University College of Medicine \\ Kang Young Lee}

Neoadjuvant chemoradiotherapy followed by total mesorectal excision has been a standard treatment for advanced rectal cancer. Preoperative chemoradiotherapy can reduce the tumor volume and reduce locoregional recurrence with complete resection of tumor. Also it facilitates function preservation surgery.

The effect of chemoradiotherapy for rectal cancer is quite variable. Complete remission was observed in about $15 \%$ of treated patients. From this result, the idea of omitting radical surgery to improve quality of life was come up and researchers have reported acceptable outcomes. However, there is still debate about how to figure out complete remission after chemoradiotherpy.

Most of clinical trials about 'wait and see' policy adapted clinical evaluation for the prediction of complete remission. Tools of clinical evaluation included rectal examination, endoscopy, and pelvic MRI. Evaluation of 
rectal mucosa with endoscopy was raised as a tool for evaluation but problem was poor sensitivity of the test. Pelvic MRI was regarded as a useful tool for re-staging after chemoradiotherapy. The sensitivity for the evaluation of circumferential margin involvement was almost $80 \%$. However, problem was that inter-observer variation was high. And the sensitivity of complete remission was still very low, for the evaluation and lymph node.

Overall sensitivity of preoperative evaluation after chemoradiotherapy is quite low, compared to one without chemoradiotherapy. The limitation of preoperative evaluation after chemoradiotherapy should be considered, when colorectal surgeons decide treatment strategy.

\section{AS2-2 How to predict the chemoradiotherapy response in rectal cancer patients}

Department of Surgery, National Defense Medical College, Tokorozawa, Japan ${ }^{1}$,

Department of Surgery, Teikyo University, Tokyo, Japan ${ }^{2)}$

Eiji Shinto $^{1)}$, Yoshiki Kajiwara ${ }^{1)}$, Takahiro Sueyama ${ }^{1)}$, Masato Yamadera ${ }^{1)}$, Tomoki Watanabe ${ }^{1)}$, Tadakazu Ao ${ }^{1)}$, Keisuke Yonemura ${ }^{1)}$, Takehiro Shiraishi ${ }^{1)}$, Ken Nagata ${ }^{1)}$, Akinori Sekizawa ${ }^{1)}$, Yojiro Hashiguchi'), Junji Yamamoto ${ }^{1)}$, Kazuo Hase ${ }^{1)}$, Hideki Ueno ${ }^{1)}$

Aim : We previously reported that increased CD8+ tumor-infiltrating lymphocytes, low tumor expression of CD133, and that of cyclooxygenase-2 (COX-2) could be useful pathological predictive markers of favorable tumor response to chemoradiotherapy (CRT) for rectal cancer (Shinto et al. Dis Colon Rect 2011 ; Ann Surg Oncol 2014). In this study, we aimed to evaluate the predictive power of the number of these three markers in two distinct cohorts.

Method : First, 95 lower rectal cancer patients who underwent preoperative short-term CRT (20 Gy in 5 fractions) followed by radical resection were retrospectively enrolled. Second, to validate the results, we prospectively recruited 40 patients who underwent long-course CRT (45 Gy in 25 fractions) followed by radical resection between 2011 and 2016. Immunohistochemical staining with antibodies for CD8, CD133, and COX -2 was performed on pretreatment biopsy specimens.

Results : In the retrospective cohort, increased CD8+ tumor-infiltrating lymphocytes negative CD133 expression, and negative COX-2 expression were associated with a favorable tumor response (area of tumor cell degeneration/necrosis $>1 / 3$ of the entire tumor lesion), respectively. When we assessed the number of these factors, the incidence of favorable tumor response was $90 \%$ in patients with 2 or 3 factors, whereas it was only $40 \%$ in patients with 0 or 1 factor $(p<0.0001)$. In addition, local recurrence rate was lower in the former group $(0 \%)$ than the latter group $(13 \% ; \mathrm{p}=0.05)$. In the prospective cohort, the predictive value of these three factors was similarly observed; i.e., there were significant differences in the incidence of marked tumor response (tumor cell degeneration/necrosis $>2 / 3$ of the entire lesion) between two groups ( $67 \%$ vs $32 \%, \mathrm{p}=0.03)$.

Conclusion : Our retrospective and prospective studies demonstrated that the combination of the number of CD8+ tumor-infiltrating lymphocytes and tumor expressions of CD133 and COX-2 could be a useful biomarker to predict the response after preoperative CRT in rectal cancer patients.

\section{AS2-3 Is induction or consolidation chemotherapy beneficial for rectal cancer following neoadjuvant chemoradiotherapy?}

Department of Surgery, Inje University, Busan Paik Hospital

Ki Beom Bae

Current Standard tri-modality therapy for rectal cancer have dramatically improved locoregional recurrence rate less than $10 \%$, but not reduce the incidence of distant recurrences, they are still consistently over $25 \%$ to $30 \%$. Distant relapse is now the main driver of adverse survival outcomes in this disease such as the high rate of liver or lung metastasis, even after a curative resection.

Although adjuvant CTx is expected to eradicate micrometastases and reduce the recurrence rate after curative resection, the long-term follow-up data from the EORTC trial showed that adjuvant FU after RT or CRT did not improve survival. By the way, the ADORE trial showed that the adjuvant administration of 
FOLFOX [oxaliplatin $(\mathrm{Ox})+5-\mathrm{FU}+$ leucovorin (LV) ] significantly improved disease-free survival (DFS) in comparison to FU/LV in stage II/III patients. However, several studies showed that approximately $50 \%$ of patients were not able to receive a full dose of a scheduled adjuvant CTx after a rectal resection, the low induction (or compliance) rates of adjuvant CTx after a rectal resection have been a serious issue.

Therefore, to maximize this adjuvant chemotherapeutic effect, the practical option could be Shifting an adjuvant CTx regimen to the preoperative period. Currently, various novel methods, such as induction CTx, consolidation CTx, concomitant administration with RT, and neoadjuvant CTx (NAC) without RT, have been trialed worldwide. These various approaches will be reviewed with recent reported clinical trials and various studies in this session.

\section{AS2-4 Pelvic lymph node dissection after neoadjuvant chemoradiotherapy \\ Cancer Institute Hospital, Tokyo, Japan \\ Takashi Akiyoshi}

Background : The necessity of lateral pelvic lymph node dissection (LLND) in patients treated with preoperative chemoradiotherapy (CRT) and the optimal treatment for suspected lateral lymph node (LLN) metastasis is controversial. In our institution, we perform selective laparoscopic LLND of the suspected side based on pretreatment imaging (more than $7 \mathrm{~mm}$ on the long-axis diameter) before CRT. The objective of this study was to evaluate the feasibility of our treatment strategy for advanced low rectal cancer.

Methods : We reviewed 369 consecutive patients with clinical stage II-III low rectal cancer who underwent curative resection between July 2004 and December 2014. The survival outcomes were compared between patients who underwent LLND (LLND group, $\mathrm{N}=124$ ) and patients who underwent total mesorectal excision alone (TME group, $\mathrm{N}=245)$.

Results : Male/female was 258/111, and the median age was 60 years (range 24-81). The median distance of the tumor from the anal verge was $40 \mathrm{~mm}$ (0-100). Laparoscopic surgery was performed in 331 patients (89.7\%), and the operative procedures were LAR/ISR/Hartmann/APR/TPE in 165/88/7/104 (28\%)/4 patients, respectively. LLND was unilateral in 102 patients (82.3\%), and LLN metastasis was confirmed in 36 patients (29\% in the LLND group). Five-year overall survival (OS), relapse-free survival (RFS), and local recurrence rate (LR) were not significantly different between TME and LLND group (OS : 88\% vs 91.2\%, $\mathrm{p}=0.6103$, RFS $: 75.5 \%$ vs $82.0 \%$, $\mathrm{p}=0.4842$, LR $: 6.1 \%$ vs $3.5 \%, \mathrm{p}=0.6254)$. Fiver-year OS, RFS, and LR were excellent $(77.8 \%, 73.8 \%, 2.9 \%$, respectively) in patients with LLN metastasis.

Conclusions : The incidence of LLN metastasis is high even after preoperative CRT, but excellent oncological outcomes can be expected in patients with LLN metastasis by the combination of CRT and selective LLND.

\section{AS2-5 TBD}

The Sixth Affiliated Hospital of Sun Yat-sen University

Yanhong Deng 